\title{
The Emission and Distribution of Dust of the Torus of NGC 1068
}

\author{
Enrique Lopez-Rodriguez ${ }^{1,2}$ (D) Lindsay Fuller $^{3}$, Almudena Alonso-Herrero ${ }^{3,4}$ (D) Andreas Efstathiou $^{5}$ (D), Kohei Ichikawa ${ }^{2,3,6}$ (ID), \\ Nancy A. Levenson ${ }^{7}$, Chris Packham ${ }^{3}$, James Radomski ${ }^{1}$, Cristina Ramos Almeida ${ }^{8,9}$ (D), Dominic J. Benford ${ }^{10}$ (D), Marc Berthoud ${ }^{11}$, \\ Ryan Hamilton $^{1}$ (D), Doyal Harper ${ }^{11}$, Attila Kováves ${ }^{12}$, Fabio P. Santos ${ }^{13}$ (D), \\ J. Staguhn ${ }^{14,15}$ (D), and Terry Herter ${ }^{16}$ (D) \\ ${ }^{1}$ SOFIA Science Center, NASA Ames Research Center, Moffett Field, CA 94035, USA; elopezrodriguez@sofia.usra.edu \\ ${ }^{2}$ National Astronomical Observatory of Japan, 2-21-1 Osawa, Mitaka, Tokyo 181-8588, Japan \\ ${ }^{3}$ Department of Physics and Astronomy, University of Texas at San Antonio, One UTSA Circle, San Antonio, TX 78249, USA \\ ${ }^{4}$ Centro de Astrobiología (CAB, CSIC-INTA), ESAC Campus, E-28692 Villanueva de la Cañada, Madrid, Spain \\ ${ }^{5}$ School of Sciences, European University Cyprus, Diogenes Street, Engomi, 1516 Nicosia, Cyprus \\ ${ }^{6}$ Department of Astronomy, Columbia University, 550 West 120th Street, New York, NY 10027, USA \\ ${ }^{7}$ Space Telescope Science Institute, 3700 San Martin Dr, Baltimore, MD 21218, USA \\ ${ }^{8}$ Instituto de Astrofísica de Canarias, C/Vía Láctea, s/n, E-38205 La Laguna, Tenerife, Spain \\ ${ }^{9}$ Departamento de Astrofísica, Universidad de La Laguna, E-38205 La Laguna, Tenerife, Spain \\ ${ }^{10}$ Goddard Space Flight Center, Greenbelt, MD 20771, USA \\ ${ }^{11}$ Yerkes Observatory, Williams Bay, WI, USA \\ ${ }^{12}$ California Institute of Technology, 301-17, 1200 East California Boulevard, Pasadena, CA 91125, USA \\ ${ }^{13}$ Center for Interdisciplinary Exploration and Research in Astrophysics (CIERA) and Department of Physics \& Astronomy, Northwestern University, \\ 2145 Sheridan Road, Evanston, IL 60208, USA \\ ${ }^{14}$ NASA Goddard Space Flight Center, Code 665, Greenbelt, MD 20771, USA \\ ${ }^{15}$ Department of Physics \& Astronomy, Johns Hopkins University, Baltimore, MD, 21218, USA \\ ${ }^{16}$ Astronomy Department, 202 Space Sciences Building, Cornell University, Ithaca, NY 14853-6801, USA \\ Received 2017 November 27; revised 2018 March 20; accepted 2018 April 9; published 2018 May 29
}

\begin{abstract}
We present observations of NGC 1068 covering the 19.7-53.0 $\mu \mathrm{m}$ wavelength range using FORCAST and HAWC + on board SOFIA. Using these observations, high-angular-resolution infrared (IR) and submillimeter observations, we find an observational turnover of the torus emission in the 30-40 $\mu \mathrm{m}$ wavelength range with a characteristic temperature of $70-100 \mathrm{~K}$. This component is clearly different from the diffuse extended emission in the narrow line and star formation regions at $10-100 \mu \mathrm{m}$ within the central $700 \mathrm{pc}$. We compute $2.2-432 \mu \mathrm{m}$ 2D images using the best inferred CLUMPY torus model based on several nuclear spectral energy distribution (SED) coverages. We find that when 1-20 $\mu \mathrm{m}$ SED is used, the inferred result gives a small torus size $(<4 \mathrm{pc}$ radius) and a steep radial dust distribution. The computed torus using the 1-432 $\mu \mathrm{m}$ SED provides comparable torus sizes, $5.1_{-0.4}^{+0.4} \mathrm{pc}$ radius, and morphology to the recently resolved $432 \mu \mathrm{m}$ Atacama Large Millimeter Array observations. This result indicates that the $1-20 \mu \mathrm{m}$ wavelength range is not able to probe the full extent of the torus. The characterization of the turnover emission of the torus using the 30-60 $\mu \mathrm{m}$ wavelength range is sensitive to the detection of cold dust in the torus. The morphology of the dust emission in our 2D image at $432 \mu \mathrm{m}$ is spatially coincident with the cloud distribution, while the morphology of the emission in the 1-20 $\mu \mathrm{m}$ wavelength range shows an elongated morphology perpendicular to the cloud distribution. We find that our 2D CLUMPY torus image at $12 \mu \mathrm{m}$ can produce comparable results to those observed using IR interferometry.
\end{abstract}

Key words: galaxies: active - galaxies: nuclei - galaxies: Seyfert - infrared: galaxies

\section{Introduction}

NGC $1068(D=14.4 \mathrm{Mpc}$, Bland-Hawthorn et al. 1997, and $1^{\prime \prime}=70 \mathrm{pc}$, adopting $H_{0}=73 \mathrm{~km} \mathrm{~s}^{-1} \mathrm{Mpc}^{-1}$ ) is the archetypical type 2 active galactic nucleus (AGN). The emission from its central engine is obscured by a distribution of optically thick dust. This dusty distribution has recently (Gallimore et al. 2016; García-Burillo et al. 2016; Imanishi et al. 2018) been resolved with the Atacama Large Millimeter Array (ALMA) observations using continuum and emission line observations with angular resolution $<0$ ". $1 \quad(<7 \mathrm{pc})$, which have provided tight constraints on the torus size and morphology. Specifically, García-Burillo et al. (2016) measured a $7-10 \mathrm{pc}$ torus diameter using $432 \mu \mathrm{m}$ continuum emission, Gallimore et al. (2016) found a $12 \times 7 \mathrm{pc}$ structure through the study of the $\mathrm{CO} J=6 \rightarrow 5$ emission, and Imanishi et al. (2018) found that both the morphology and dynamics of the $\mathrm{HCN} J=3 \rightarrow 2$ and $\mathrm{HCO}^{+} J=3 \rightarrow 2$ emission are fairly aligned in the east-west direction with a size of $\sim 12 \times$ 5 pc. In addition, infrared (IR) interferometric observations (Wittkowski et al. 2004; Jaffe et al. 2004; Raban et al. 2009; López-Gonzaga et al. 2014) have put tight constraints on the different emission components of the torus in NGC 1068. Specifically, a $700 \mathrm{~K}$ dust structure with a size $1.4 \mathrm{pc}, 250 \mathrm{~K}$ structure with a size of $3 \mathrm{pc}$, and an extended $14 \mathrm{pc}$ dust component in the polar direction with a characteristic temperature of $350 \mathrm{~K}$. These observations have challenged our current understanding on the emission and distribution of dust surrounding the active nucleus of NGC 1068.

The torus is not resolved by the current suite of single-dish telescopes, thus spectral energy distribution (SED) modeling using the best angular resolution to isolate the torus emission from extended diffuse dust emission, star formation regions, and/or host galaxy is crucial to obtain physical information about the torus (i.e., Mason et al. 2006; Hönig et al. 2008; 
Alonso-Herrero et al. 2011; Ramos Almeida et al. 2011, 2014; Feltre et al. 2012; Ichikawa et al. 2015). These extensive works have been performed in the $1-20 \mu \mathrm{m}$ wavelength range with the general agreement that the torus is formed by a clumpy distribution of optically thick dust with sizes of a fews parsec surrounding the central engine. The 1-20 $\mu \mathrm{m}$ high-angular resolution observations show an increase in the total flux density with increasing wavelength. The silicate feature at 10 and $18 \mu \mathrm{m}$, the near-IR (NIR) emission, and the luminosity of the torus in the $10 \mu \mathrm{m}$ window provide important diagnostic tools to constrain the torus structure (Ramos Almeida \& Ricci 2017). However, these studies show that the turnover of the torus emission occurs in the 20-30 $\mu \mathrm{m}$, which causes the torus emission to be dominated by warm dust with a characteristic temperature of $\sim 100-150 \mathrm{~K}$, and with typical torus diameters of $\leqslant 5 \mathrm{pc}$-slightly smaller than the currently resolved observations by ALMA.

There is an observational gap within the $20-70 \mu \mathrm{m}$ wavelength range with angular resolutions $<10^{\prime \prime}$, where warm/cold dust in the torus seems to have its peak of emission, and that it has not been characterized. A Bayesian exploration study by Asensio Ramos \& Ramos Almeida (2013) using CLUMPY torus models found that the region between 10 and $200 \mu \mathrm{m}$ provides the best wavelength range to constrain the torus radial extent and the number and radial distribution of clouds in the torus. Fuller et al. (2016) observationally show the potential of far-IR (FIR) observations to constrain the torus size using $31.5 \mu \mathrm{m}$ imaging observations with the Faint Object Infrared Camera for the SOFIA Telescope (FORCAST) on board SOFIA. They found that (1) the torus radial extent model parameter decreases by $30 \%$ in size for $60 \%$ (6 out of 10 AGNs) of their sample, and (2) the SED turnover of the torus emission does not occur up to $31.5 \mu \mathrm{m}$, in $F_{\nu}$. Their observations also show resolved diffuse extended emission along the narrow line region (NLR), which allowed them to better isolate the torus emission using SOFIA than previous Spitzer $30-40 \mu \mathrm{m}$ observations. The combination of fully sampled nuclear SED, resolved IR interferometric and ALMA observations, and torus models is crucial to break degeneracies in the physical properties of the torus.

With the tight constraint on the torus size of NGC 1068 provided by the resolved images by ALMA, and the currently available moderate angular resolution FIR capabilities, we here present an observational study to characterize the emission and distribution of dust through the characterization of the SED of NGC 1068 using torus models. We present observations of NGC 1068 covering the 19.7-37.1 $\mu \mathrm{m}$ wavelength range using FORCAST and newly obtained $53.0 \mu \mathrm{m}$ imaging observations by the High-resolution Airborne Wideband Camera (HAWC+) on board SOFIA. The paper is organized as follows: Section 2 describes the observations and data reduction, Section 3 discusses the emission and dust distribution of dust in the torus of NGC 1068, and Section 4 shows the spectral decomposition of the nuclear SED. In Section 5, we present our conclusions.

\section{Observations and Data Reduction}

\subsection{FORCAST Observations}

NGC 1068 was observed as part of the Guaranteed Time Observations (GTO; PI: T. Herter) on 2016 September 17 using

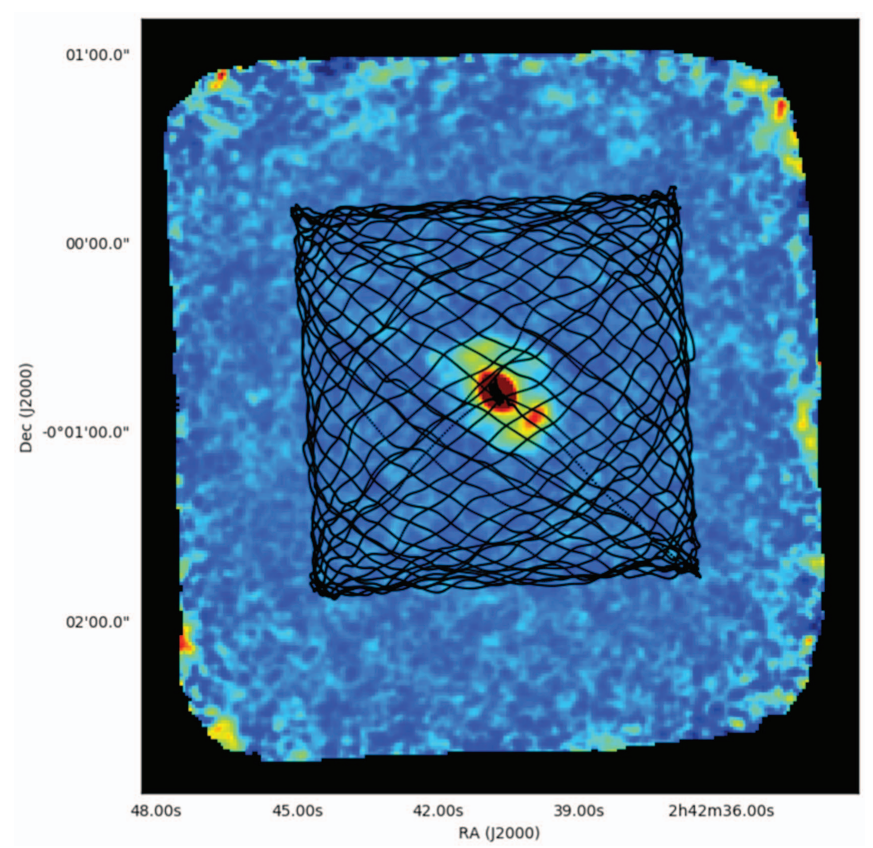

Figure 1. Example of a single Lissajous pattern (black line) overlaid on the final image at $53 \mu \mathrm{m}$ of NGC 1068 (color scale) using several Lissajous scans.

FORCAST (Herter et al. 2012) on the $2.5 \mathrm{~m}$ SOFIA telescope. We made observations with the dual-channel mode at the 19.7, 31.5 , and $37.1 \mu \mathrm{m}$ using the two-position chop-nod $(\mathrm{C} 2 \mathrm{~N})$ method with symmetric nod-match-chop to remove timevariable sky background and telescope thermal emission and to reduce the effect of $1 / f$ noise from the array. In all observations, we used an instrumental position angle, i.e., long axis of the detector with respect to the north on the sky, of $305^{\circ}$, a chop-throw of $1^{\prime}$ with a $30^{\circ} \mathrm{E}$ of $\mathrm{N}$ chop-angle. The onsource times were $427 \mathrm{~s}, 471 \mathrm{~s}$, and $343 \mathrm{~s}$ at $19.7 \mu \mathrm{m}, 31.5 \mu \mathrm{m}$, and $37.1 \mu \mathrm{m}$, respectively.

SOFIA provided reduced data using the FORCAST REDUX PIPELINE V1.1.3 following the method described by Herter et al. (2013) to correct for bad pixels, "droop" effect, nonlinearity, and cross-talk. The point-spread functions (PSFs) of the observations were estimated using observations of Ceres taken immediately before NGC 1068 observations with the same instrumental configuration and bands. We estimated a full width at half maximum (FWHM) of Ceres of $2.4,2$." 8 , and 2.9 at $19.7 \mu \mathrm{m}, 31.5 \mu \mathrm{m}$, and $37.1 \mu \mathrm{m}$, respectively. NGC 1068 was flux-calibrated using the set of standard stars of the observing run, which provides flux uncertainties of $5.0 \%$, $5.2 \%$, and $7.7 \%$ at $19.7 \mu \mathrm{m}, \quad 31.5 \mu \mathrm{m}$, and $37.1 \mu \mathrm{m}$, respectively.

\section{2. $H A W C+$ Observations}

NGC 1068 was observed as part of the GTO (PI: D. Dowell) on 2017 May 06 using HAWC+ (Vaillancourt et al. 2007; D. A. Harper et al. 2018, in preparation) on the $2.5 \mathrm{~m}$ SOFIA telescope. We made observations using the Lissajous pattern in the total intensity mode at $53 \mu \mathrm{m}\left(\lambda_{c}=53 \mu \mathrm{m}, \Delta \lambda / \lambda_{c}=0.17\right.$ bandwidth). In this new SOFIA observing mode, the telescope is driven to follow a parametric curve at a nonrepeating period whose shape is characterized by the relative phases and frequency of the motion. Figure 1 shows the Lissajous pattern 
of a single observation at $53 \mu \mathrm{m}$ of NGC 1068 with a scan rate of $100^{\prime \prime} \mathrm{s}^{-1}$ and a $60^{\prime \prime}$ scan amplitude. We performed a total of five Lissajous scans with relative phases of $5^{\circ}$ and $27^{\circ}$ with a total on-source time of $455 \mathrm{~s}$.

We reduced the data using the Comprehensive Reduction Utility for SHARC II v2.34-3beta (CRUSH, Kovács 2006, 2008) ${ }^{17}$ optimized for HAWC + and the HAWC_DPR PIPELINE V1.1.1. CRUSH estimates and removes the correlated atmospheric and instrumental signals, solves for the relative detector gains, and determines the noise weighting of the time streams in an iterated pipeline scheme. The PSF was estimated using Uranus observations on 2017 May 07 with an FWHM of 4!" 9, consistent with diffraction-limited observations at $53 \mu \mathrm{m}$. Flux calibrators were not observed during the same flight, thus we crosscalibrated our observations using flux calibrators, i.e., Uranus, from other flights. Although we find a flux calibration accuracy of $\sim 8 \%$ for observations taken within the same flight, the crosscalibration between flights can only ensure a flux accuracy of $\sim 20 \%$.

\subsection{Photometry and Nuclear Flux Imaging Modeling}

We aim to obtain the emission from the unresolved core of NGC 1068 at all the observed wavelengths. The SOFIA observations of NGC 1068 show a resolved core (Figure 2) that is thought to arise from an unresolved component and an extended component. To obtain the fractional contribution to the total emission from both unresolved and extended components, we made two different photometric measurements (Table 1). First, the flux in a circular aperture of $10^{\prime \prime}(700 \mathrm{pc})$ diameter was measured, which ensures to enclose the whole flux of an unresolved source at the given wavelength and minimizes the contribution from the diffuse extended emission. Second, the central $20^{\prime \prime} \times 20^{\prime \prime}\left(1.4 \times 1.4 \mathrm{kpc}^{2}\right)$ emission was fitted with a composite model using the corresponding PSF to each observation and a 2D Gaussian profile. We refer to these methods as "aperture" and "PSF-scaling" photometry, respectively, in the remainder of the paper. The aperture photometry represents the total flux from the observed galaxy at a given wavelength, $F_{T}$. In the PSF-scaling method, the total flux from the scaled-PSF, $F_{\mathrm{PSF}}$, represents the maximum likely contribution from an unresolved nuclear component at the given angular resolution of the observations, while the total flux from the 2D Gaussian profile, $F_{\text {ext }}$, provides the minimum contribution of the extended component surrounding the central source. We estimated the total flux of the model, $F_{T}^{M}$, as the sum of both the PSF and the 2D Gaussian profile. The PSFscaling method has five free parameters, the amplitudes of both PSF and 2D Gaussian profile, the FWHM of the long, $b$, and short, $a$, axes and the position angle (P.A.) of the 2D Gaussian profile. The fitting routine minimizes the residuals (galaxy minus model: scaled-PSF $+2 \mathrm{D}$ Gaussian) to a level $<5 \%$ of the total flux, $F_{T}$, within the central $20^{\prime \prime}$ diameter. We also considered a 2D Sérsic profile to fit the extended component. We obtained index profiles $\sim 0.5$ and size parameters of the Sérsic profiles similar to the FWHM of the observations, which is close to the special case of the Sérsic profile tending to a Gaussian profile. Due to this behavior and that the Sérsic profile increases the number of free parameters, we decided to use 2D Gaussian profiles.

\footnotetext{
${ }^{17}$ CRUSH can be found at http://www.submm.caltech.edu/ sharc/crush/.
}

The uncertainty in the photometry was estimated in the following manner. The aperture photometry uses the flux uncertainties estimated from the flux calibration described in Sections 2.1 and 2.2. For the PSF-scaling photometry, an estimate of the error induced by a variable PSF was obtained by cross-calibrating the standard stars observed on the same or several nights. This error was found to be $\sim 5 \%$. Another estimate of the error induced by the fitting procedure was estimated to be $\sim 3 \%$. The total uncertainty for the PSF-scaling photometry was calculated by adding in quadrature these individual contributions.

Figure 2 shows the NGC 1068 observations, scaled PSF, Model (PSF+2D Gaussian), and residuals (NGC 1068-Model) of the central $50^{\prime \prime} \times 50^{\prime \prime}\left(3.5 \times 3.5 \mathrm{kpc}^{2}\right)$ observations at 19.7, $31.5,37.1$, and $53 \mu \mathrm{m}$. Table 1 shows the measured and modeled nuclear photometry for each photometric method and model component in the central $10^{\prime \prime}$ aperture. The fractional contribution of the PSF and 2D Gaussian profile in the central $10^{\prime \prime}$ diameter is also shown. Our flux density at $19.7 \mu \mathrm{m}$ estimated by using PSF-scaling of $22.0 \pm 1.4 \mathrm{Jy}$ is in excellent agreement with the flux density of $20.2 \pm 3.4 \mathrm{Jy}$ in a 0 ". 4 aperture by Tomono et al. (2001). We took PACS/Herschel spectroscopic data of NGC 1068 from the Herschel Archive and we obtained a nuclear flux density of $\sim 70 \mathrm{Jy}$ at $60 \mu \mathrm{m}$ per spaxel, where a spaxel is 9!. $4 \times 9$ !. $4\left(658 \times 658 \mathrm{pc}^{2}\right)$. Despite the difference in wavelength, this result is in good agreement with our total flux of $72 \pm 14 \mathrm{Jy}$ at $53 \mu \mathrm{m}$ using HAWC + . At all wavelengths, the total flux model, $F_{T}^{M}$, is $<2 \%$ of the total flux of the observations, $F_{T}$, and the P.A. of the extended emission is $44.7 \pm 1.3$ with a decrease of the ratio of the short and long axis, $a / b$, from 0.85 at $19.7 \mu \mathrm{m}$ to 0.61 at $53 \mu \mathrm{m}$. Our extended diffuse emission is spatially coincident with the large scale, $32^{\prime \prime}(1.92 \mathrm{kpc})$ inner bar at a P.A. of $48^{\circ} \pm 3^{\circ}$, the socalled NIR bar (Scoville et al. 1988; Schinnerer et al. 2000; Emsellem et al. 2006). Despite any contribution of diffuse extended emission within the PSF of SOFIA in the $20-53 \mu \mathrm{m}$ wavelength range (Section 4), we found a turnover of the unresolved emission, $F_{\mathrm{PSF}}$ in Table 1 , within the $31.5-53 \mu \mathrm{m}$ wavelength range. Specifically, the PSF fractional contribution to the total flux decreases from $\sim 50 \%$ in the $30-40 \mu \mathrm{m}$ wavelength range to $<40 \%$ at shorter and longer wavelengths. This result is in agreement with the observational constraint by Fuller et al. (2016), who suggested that the turnover of the torus emission does not occur until wavelengths $>31.5 \mu \mathrm{m}$ for a sample of nearby AGNs.

\section{The Torus of NGC 1068}

\subsection{CLUMPY Torus Models}

We here describe the details of the fitting to the nuclear SED of NGC 1068. The nuclear SED is composed of our PSFscaling photometry $\left(F_{\mathrm{PSF}}\right)$ in conjunction with the 0 ". 4 aperture photometry from 2 to $20 \mu \mathrm{m}$ photometry by Tomono et al. (2001), the $8-13 \mu \mathrm{m}$ nuclear spectrum in a 0 ". 4 aperture using Michelle on the $8.1 \mathrm{~m}$ Gemini-north Telescope by Mason et al. (2006), and the $432 \mu \mathrm{m}$ ALMA observation by García-Burillo et al. (2016). We fitted the nuclear SED using the CLUMPY torus models of Nenkova et al. (2008) and the BAYESCLUMPY approach developed by Asensio Ramos \& Ramos Almeida (2009). This approach has been successfully applied to this and other Seyfert galaxies (e.g., Alonso-Herrero et al. 2011; Ichikawa et al. 2015). The free parameters of the model were 

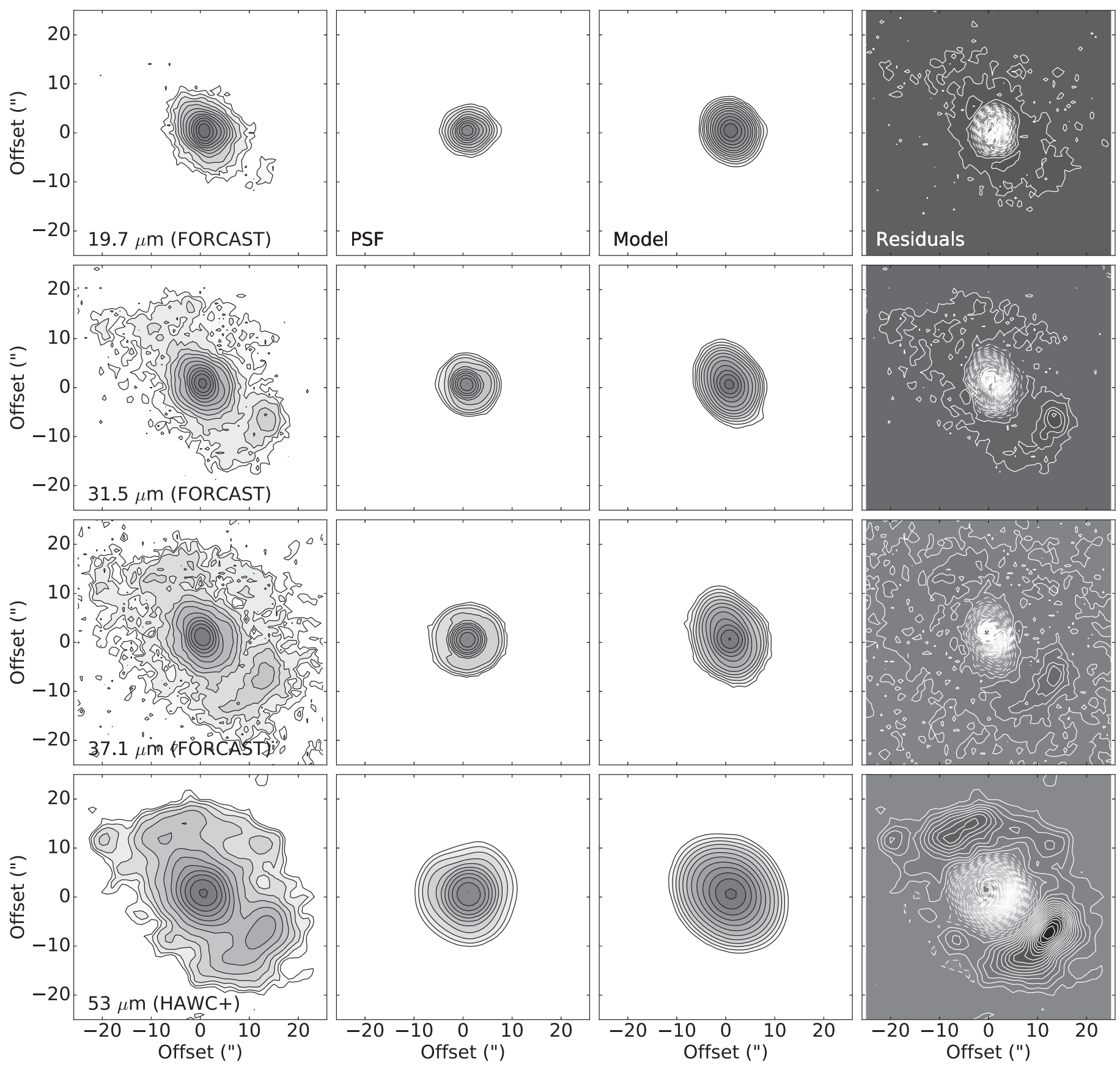

Figure 2. From left to right: NGC 1068 observations, scaled PSF, Model (PSF+2D Gaussian) of the central $20^{\prime \prime} \times 20^{\prime \prime}\left(1.4 \times 1.4 \mathrm{kpc}^{2}\right)$, and Residuals (NGC 1068 Model) at $19.7 \mu \mathrm{m}, 31.5 \mu \mathrm{m}, 37.1 \mu \mathrm{m}$ and $53 \mu \mathrm{m}$ from top to bottom, respectively. In all cases, the FOV is $50^{\prime \prime} \times 50^{\prime \prime}\left(3.5 \times 3.5 \mathrm{kpc}^{2}\right)$. NGC 1068 , PSF and Model contours are shown in $\log$ (flux density [Jy]) from -2.0 to 1.5 in steps of 0.2 . Residual contours are shown in flux density (Jy) from $-0.4 \mathrm{Jy}$ to $0.3 \mathrm{Jy}$ in steps of $0.02 \mathrm{Jy}$. North is up and east is left.

set with a flat prior distribution, with the exception of the foreground visual extinction to the core, $A_{V}$, was set to be in the $0-10 \mathrm{mag}$ range. The best inferred model with the $1 \sigma$ uncertainty region is shown in Figure 3, and the posterior distributions of each model parameter are shown in the Appendix. Table 2 shows the output values of each torus model parameter.

In general, the full family of CLUMPY torus model solutions when using the SOFIA observations from 20 to $53 \mu \mathrm{m}$ provides a tighter $1 \sigma$ dispersion than previous studies (i.e., Alonso-Herrero et al. 2011, Figure 5; García-Burillo et al. 2016, Figure 4, our Figure 4). This result is due to the better sampling of the turnover of the torus emission in the $30-40 \mu \mathrm{m}$ as in comparison with previous studies. The median value for the foreground visual extinction was found to be $A_{V}=9_{-1}^{+1}$ mag. We notice that if the extinction to the core was set to be negligible, $A_{V}<5 \mathrm{mag}$, then the fitting tends to obtain viewing angles, $i$, of the torus in the $30^{\circ}-40^{\circ}$ range, and does not fit the SED in the $1-5 \mu \mathrm{m}$ wavelength range. Packham et al. (1997) found that a visual extinction of $36 \mathrm{mag}$ to the core of NGC 1068 can explain the 


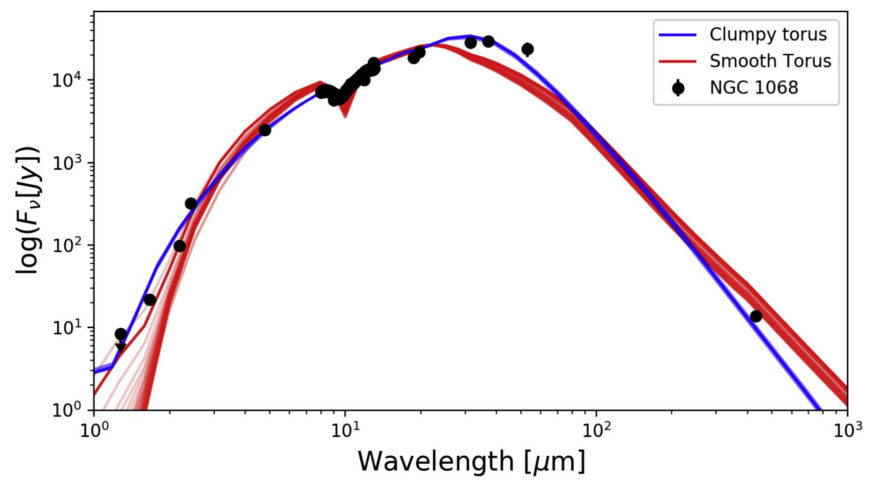

Figure 3. Best-fit (solid line) and $1 \sigma$ uncertainties (shadowed area) of the CLUMPY (blue) and smooth (red) torus models to the nuclear SED (black dots) of NGC 1068.

absorptive polarization at $2.0 \mu \mathrm{m}$, compatible with the expected null polarization observed at $10 \mu \mathrm{m}$ by the emissive polarization of the torus found by Lopez-Rodriguez et al. (2016). Both works also found a visual extinction by the central dust lane to be $\sim 8$ mag. Thus, we expect that the torus emission is extinguished by a column of dust into our LOS with a visual extinction in the range of 8-36 mag, our computed visual extinction of $A_{V}=9_{-1}^{+1}$ mag is in agreement within that range. We obtained a viewing angle, $i=75_{-4}^{+8}$ o compatible with the $\mathrm{H}_{2} \mathrm{O}$ maser observations in the central parsec of NGC 1068, which suggests a torus with an almost edge-on view, $\sim 90^{\circ}$.

Based on the best inferred CLUMPY torus model, we can estimate torus morphological parameters as the outer radius, $r_{\text {out }}=r_{\text {in }} Y$ pc, where $r_{\text {in }}$ is the inner radius of the torus defined by the distance of the sublimation temperature of dust grains, $T$, as a function of the bolometric luminosity, $L_{\mathrm{bol}}$, as $r_{\mathrm{in}}=$ $0.4\left(L_{\mathrm{bol}} / 10^{45} \mathrm{erg} \mathrm{s}^{-1}\right)^{0.5}(T / 1500 \mathrm{~K}) \mathrm{pc}$ (Barvainis 1987), and the torus scale height as $H=r_{\text {out }} \sin \sigma$ pc. The estimated bolometric luminosity from our CLUMPY torus model, $L_{\mathrm{bol}}=$ $5.02_{-0.15}^{+0.19} \times 10^{44} \mathrm{erg} \mathrm{s}^{-1}$, yields an inner torus radius of $r_{\text {in }}=$ $0.28_{-0.01}^{+0.01} \mathrm{pc}$ for dust grains at a temperature of $1500 \mathrm{~K}$. Using $Y=18_{-1}^{+1}$ and $\sigma=43_{-15}^{+12 \circ}$, the torus radius and scale height are estimated to be $r_{\text {out }}=5.1_{-0.4}^{+0.4} \mathrm{pc}$ and $H=3.5_{-1.3}^{+1.0} \mathrm{pc}$, respectively. Table 2 summarizes these results. These results are in agreement with the resolved $7-10 \mathrm{pc}$ torus extension (García-Burillo et al. 2016), $12 \times 7$ pc (Gallimore et al. 2016), and $12 \times 5$ pc (Imanishi et al. 2018) torus diameter using ALMA observations. We can estimate the visual extinction into our LOS, $A_{\mathrm{v}}^{\mathrm{LOS}}$, as $A_{\mathrm{v}}^{\mathrm{LOS}}=1.086 N_{0} \tau_{\mathrm{v}} \exp \left(-(i-90)^{2} / \sigma^{2}\right)$ mag. From our best CLUMPY torus model, we estimate $A_{\mathrm{v}}^{\text {LOS }}=$ $248_{-142}^{+201}$ mag. Using the standard Galactic ratio $A_{\mathrm{v}} / N_{\mathrm{H}}=5.23 \times$ $10^{-22}$ mag cm$~^{2}$ (Bohlin et al. 1978), we estimate a column density of $N_{\mathrm{H}}=4.7_{-2.7}^{+3.9} \times 10^{23} \mathrm{~cm}^{-2}$.

\subsection{Smooth Torus Models}

We have also used smooth torus models (Efstathiou \& Rowan-Robinson 1995) to fit the nuclear SED of NGC 1068. The best-fit model and the output parameters are shown in Figure 3 and Table 2, respectively. In general, the smooth torus models reproduce well the nuclear SED of NGC 1068, except for the FIR $(20-60 \mu \mathrm{m})$ wavelength range. In this spectral range, the smooth torus models underestimate the measured nuclear fluxes. We note that if we force these models to fit the FIR range, then the smooth torus models overpredict the

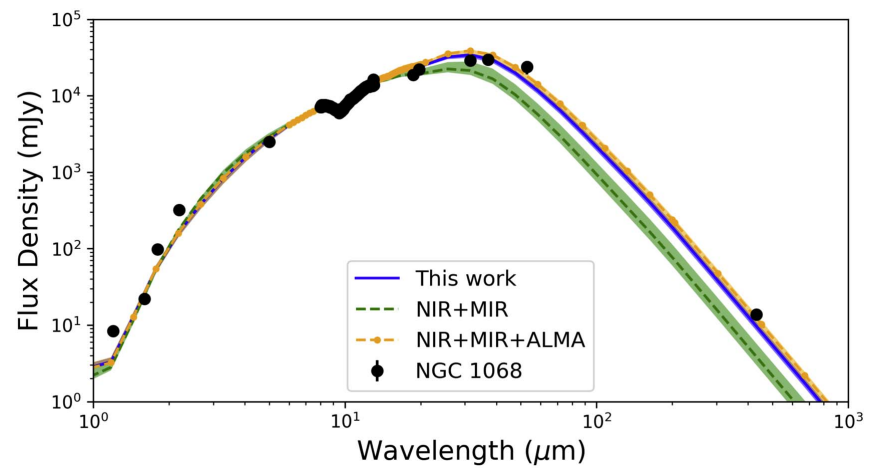

Figure 4. CLUMPY torus models inferred using different SED sampling (Table 3). The posterior distributions for each model are shown in Table 3 and Figure 7.

submillimeter fluxes by a factor of 10 or more, and the torus size increases to a few tens of parsecs. Thus, we use the MIR spectroscopic observations, i.e., the $10 \mu \mathrm{m}$ silicate feature, and the ALMA observations to find the best fit of the smooth torus model.

We find a smooth torus with similar physical characteristics as the CLUMPY torus (Table 2), except for the outer radius, which is larger in the case of the smooth torus. This difference is mainly due to the sublimation temperature used by both models, the smooth torus models use a maximum temperature of dust grains of $1000 \mathrm{~K}$, in comparison with the $1500 \mathrm{~K}$ used by the CLUMPY torus models. Although we exclusively used the smooth torus models of Efstathiou \& Rowan-Robinson (1995), we speculate that other smooth torus models (i.e., Schartmann et al. 2005; Fritz et al. 2006) may similarly fail to account for the complete SED of the torus. This may be due to the fact that smooth models generally have much less flexibility in the specification of the distribution of the dust in the torus and especially its outer part to which the far-infrared and submillimeter observations are more sensitive. This certainly merits further study and we plan to pursue this in future work.

\subsection{The CLUMPY Torus Properties versus SED Coverage}

We here investigate the emission and distribution of dust in the torus of NGC 1068 using several SED coverages. Direct comparison between previous studies are not straightforward due to the development of the CLUMPY torus models through the past several years. ${ }^{18}$ Our nuclear SED is constructed using the mentioned previous studies in Section 3.1. Thus, to avoid any potential misinterpretation of the physics of the torus that a slightly different version of the models could introduce, we here reanalyze the nuclear SED as a function of the SED coverage using the most updated version of the CLUMPY torus models. Table 3 shows the output parameters of the best inferred CLUMPY torus model for an SED using 1-20 $\mu \mathrm{m}$ imaging and spectroscopic observations (labeled as NIR $+\mathrm{MIR})$, and for an SED using 1-20 $\mu \mathrm{m}$ imaging and spectroscopic observations and ALMA observations (labeled as NIR+MIR+ALMA). The posterior distributions for each parameter with their median value and $1 \sigma$ error are shown in the Appendix. For each SED coverage, Figure 4 shows the best inferred CLUMPY torus model and their $1 \sigma$ uncertainty.

\footnotetext{
${ }^{18}$ News update of CLUMPY torus models: https://www.clumpy.org/pages/ news-updates.html.
} 
Table 1

Measured and Modeled Nuclear Photometry

\begin{tabular}{|c|c|c|c|c|c|c|c|c|c|}
\hline \multicolumn{7}{|c|}{ Photometric Measurements $^{\mathrm{a}}$} & \multicolumn{3}{|c|}{ Spectral Decomposition $^{\mathrm{b}}$} \\
\hline $\begin{array}{l}\lambda_{c} \\
(\mu \mathrm{m})\end{array}$ & $\begin{array}{c}F_{T} \\
(\mathrm{Jy})\end{array}$ & $\begin{array}{l}F_{T}^{M} \\
(\mathrm{Jy})\end{array}$ & $\begin{array}{l}F_{\mathrm{PSF}} \\
(\mathrm{Jy})\end{array}$ & $\begin{array}{l}F_{\text {ext }} \\
(\mathrm{Jy})\end{array}$ & $\begin{array}{l}\mathrm{PSF} \\
\%\end{array}$ & $\begin{array}{c}\text { Extended } \\
\%\end{array}$ & $\begin{array}{c}\text { Star Formation } \\
\%\end{array}$ & $\begin{array}{c}\text { Dust at } 200 \mathrm{~K} \\
\%\end{array}$ & $\begin{array}{c}\text { Torus } \\
\%\end{array}$ \\
\hline 19.7 & $61.9 \pm 3.1$ & $60.9 \pm 3.8$ & $22.0 \pm 1.4$ & $38.9 \pm 2.4$ & $36 \pm 4$ & $64 \pm 7$ & 10 & 60 & 30 \\
\hline 31.5 & $59.4 \pm 3.1$ & $58.4 \pm 3.7$ & $28.8 \pm 1.8$ & $29.6 \pm 1.9$ & $49 \pm 5$ & $51 \pm 6$ & 23 & 36 & 41 \\
\hline 37.1 & $59.6 \pm 4.6$ & $58.8 \pm 5.0$ & $29.7 \pm 2.5$ & $29.1 \pm 2.5$ & $51 \pm 8$ & $49 \pm 8$ & 33 & 26 & 41 \\
\hline 53.0 & $71.6 \pm 14.3$ & $71.5 \pm 14.5$ & $23.8 \pm 4.8$ & $47.7 \pm 9.7$ & $33 \pm 15$ & $67 \pm 25$ & 64 & 16 & 20 \\
\hline
\end{tabular}

Notes. Fractional contribution of emissive components in a $10^{\prime \prime}$ aperture.

${ }^{\mathrm{a}}$ Measured and modeled photometry as described in Section 2.3.

${ }^{\mathrm{b}}$ Fractional contribution of the several components used in the spectral decomposition described in Section 4 . We estimate a $5 \%$ uncertainty for the fractional contribution of each component.

Table 2

CLUMPY and Smooth Torus Model Parameters

\begin{tabular}{|c|c|c|c|c|c|}
\hline \multicolumn{3}{|c|}{ CLUMPY Torus } & \multicolumn{3}{|c|}{ Smooth Torus } \\
\hline Parameter & Symbol & Value & Parameter & Symbol & Value \\
\hline Angular width & $\sigma$ & $43_{-15}^{+12 \circ}$ & Opening angle & $\theta_{O A}$ & $37_{-8}^{+230}$ \\
\hline Number clouds along the equatorial plane & $N_{0}$ & $4_{-1}^{+2}$ & $\cdots$ & $\ldots$ & $\cdots$ \\
\hline Index of the radial density profile & $q$ & $0.08_{-0.06}^{+0.19}$ & Index of the radial density profile & $q_{s}$ & 1 (fixed) \\
\hline Optical depth of each cloud & $\tau_{v}$ & $70_{-14}^{+6}$ & Optical depth of the torus, LOS & $\tau_{v, s}$ & $250_{-10}^{+20}$ \\
\hline Inner radius & $r_{\text {in }}$ & $0.28_{-0.01}^{+0.01} \mathrm{pc}$ & & $r_{\mathrm{in}, s}$ & $0.41_{-0.02}^{+0.05} \mathrm{pc}$ \\
\hline Outer radius & $r_{\text {out }}$ & $5.1_{-0.4}^{+0.4} \mathrm{pc}$ & & $r_{\text {out }, s}$ & $8.5_{-0.7}^{+7.9} \mathrm{pc}$ \\
\hline Height & $H$ & $3.5_{-1.3}^{+1.0} \mathrm{pc}$ & & $H_{s}$ & $4.2_{-0.2}^{+0.5} \mathrm{pc}$ \\
\hline Bolometric luminosity ( $\mathrm{erg} \mathrm{s}^{-1}$ ) & $L_{\mathrm{bol}}$ & $5.02_{-0.19}^{+0.15} \times 10^{44}$ & & $L_{\mathrm{bol}, s}$ & $1.11_{-1.23}^{+0.28} \times 10^{44}$ \\
\hline
\end{tabular}

When CLUMPY torus model fitting is used with data only in the $1-20 \mu \mathrm{m}$ wavelength range, (1) the torus is smaller and more compact (large $q$ values) than the current resolved torus of $\sim 10$ pc diameter of NGC 1068 by the ALMA observations, and (2) the turnover of the torus emission peaks at shorter wavelengths than when the SED coverage includes observations at longer wavelengths. This result implies that $1-20 \mu \mathrm{m}$ observations are not able to probe the full extent of the torus. Despite the angular resolution, 2."4-4."9, Figure 4 shows that the turnover of the torus emission occurs in the range of $30-40 \mu \mathrm{m}$, which corresponds to a characteristic temperature of $70-100 \mathrm{~K}$. This result indicates that (1) the amount of cold dust and/or (2) the radiation from indirectly radiated clouds is substantial to shift the peak emission of the torus toward longer wavelengths.

\subsection{D CLUMPY Torus Images}

We use the radiative transfer code CLUMPY torus (Nenkova et al. 2008) to compute the surface brightness and cloud distributions of the dusty torus as a function of wavelength for each set of parameters shown in Table 3. Specifically, we use the HyperCubes of AGN Tori (HYPERCAT ${ }^{19}$; R. Nikutta et al. 2018, in preparation). HYPERCAT uses the CLUMPY torus models with any combination of parameters to generate physically scaled and flux-calibrated 2D images of the dust

\footnotetext{
19 CLUMPY images can be found at https://www.clumpy.org/pages/images. html.
}

emission and distribution for a given AGN. We use a distance of $14.4 \mathrm{Mpc}$, a torus orientation on the plane of the sky of $\sim 138^{\circ}$ east of north based on the IR polarimetric signature of the nucleus (e.g., Packham et al. 1997; Simpson et al. 2002; Gratadour et al. 2015; Lopez-Rodriguez et al. 2015), and the bolometric luminosities and model parameters by each inferred model from Table 3. Figure 5 shows the dust emission distribution from 2.2 to $432 \mu \mathrm{m}$ for the $1-20 \mu \mathrm{m}$ SED+MIR Spectroscopy (labeled as NIR+MIR), 1-20 $\mu \mathrm{m}$ SED+MIR Spectroscopy+ALMA (labeled as NIR+MIR+ALMA), and this work, whose torus parameters are summarized in Table 2. The last column shows the cloud distribution for each CLUMPY torus.

For all cases, the clouds are distributed in the equatorial plane with major differences in their torus sizes, angular widths, and radial density profile (Figure 4, last column). These differences affect the morphology of the dust emission as a function of wavelength. We find that the $2.2 \mu \mathrm{m}$ dust emission is concentrated on the inner edge of the torus where dust is directly radiated by the central engine, while the $8-12 \mu \mathrm{m}$ dust emission is along the polar direction as the high opacity in the equatorial direction is absorbing most of the radiation from the central engine. At longer wavelengths, $>30 \mu \mathrm{m}$, the dust emission is along the equatorial plane, where the $432 \mu \mathrm{m}$ truly describes the bulk of dust distribution in the torus.

We point out the tight morphological and size similarities between our 2D CLUMPY torus image at $432 \mu \mathrm{m}$, using the well sampled SED from 1 to $432 \mu \mathrm{m}$, with the observed torus emission by the ALMA observations (García-Burillo et al. 2016; 

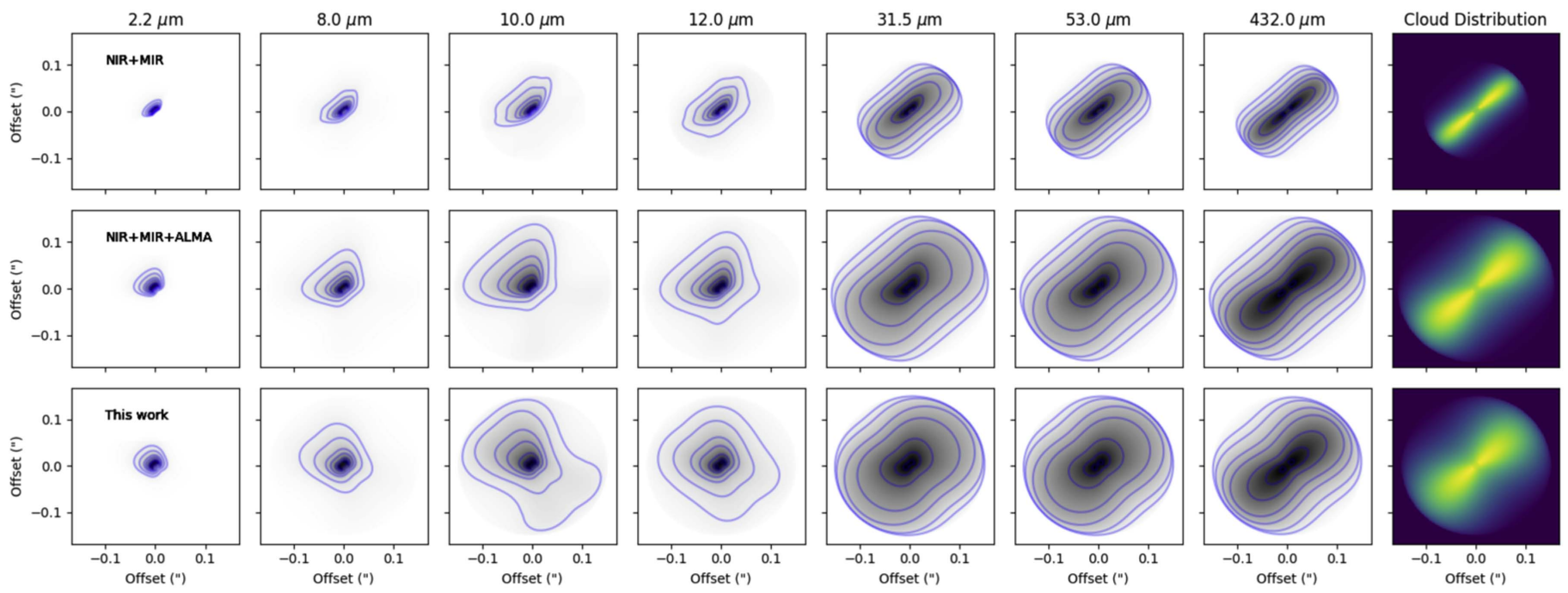

Fure 5. 2D CLUMPY torus images of NGC 1068 generated using HyperCAT based on the several SEDs in Table 3 as a function of wavelength. The first seven columns show the dust emission from 2.2 to $432 \mu \mathrm{m}$, while the last column shows the cloud distribution. Contours show the intensities at the levels of 0.05 , $0.1,0.2$, $0.3,0.5,0.7$, and 0.9 times the peak flux. In all cases, the model was scaled to a distance of $14.4 \mathrm{Mpc}$; north is up and east is left.

Table 3

CLUMPY Torus Model Parameters As a Function of SED Coverage

\begin{tabular}{lccccccc}
\hline \hline$\sigma\left(^{\circ}\right)$ & $Y$ & $N_{0}$ & $q$ & $\tau_{v}$ & $i\left(^{\circ}\right)$ & $r_{\text {out }}(\mathrm{pc})$ & SED \\
\hline $20_{-3}^{+5}$ & $13_{-3}^{+4}$ & $11_{-3}^{+2}$ & $0.22_{-0.13}^{+0.20}$ & $28_{-6}^{+10}$ & $75_{-6}^{+4}$ & $3.5_{-0.9}^{+1.3}$ & $1-20 \mu \mathrm{m}$ SED+MIR Spectroscopy \\
$31_{-8}^{+20}$ & $19_{-1}^{+1}$ & $5_{-2}^{+3}$ & $0.06_{-0.04}^{+0.08}$ & $59_{-13}^{+16}$ & $71_{-3}^{+5}$ & $5.5_{-0.4}^{+0.4}$ & $1-20 \mu \mathrm{m}$ SED+MIR Spectroscopy+ALMA \\
\hline
\end{tabular}

Imanishi et al. 2018). However, when ALMA observations are compared with the $2 \mathrm{D}$ images produced by the $1-20 \mu \mathrm{m}$ SED, the inferred torus model is smaller, more compact, and thinner, which supports the discussion above regarding the $1-20 \mu \mathrm{m}$ observations, which underestimate the true size of the torus.

Using our 2D CLUMPY torus image at $12.0 \mu \mathrm{m}$, we find that the dust emission along the polar direction is $\sim 5$ times that of the central dust emission. We estimate the dust emission along the polar direction using an ellipse of semimajor axis of 139 mas ( $9.7 \mathrm{pc}$ ) and an eccentricity of 0.91 , while the central dust emission was estimated in a circular aperture of 50 mas $(3.5 \mathrm{pc})$ diameter. At this wavelength, the north emission is more prominent than that coming from the south, due to the inferred torus inclination of $75^{\circ}$. We estimate that the fractional contribution along the polar direction increases at larger inclinations, although further extinction by the host galaxy and/or dust in the NLR will play an important role in the observed emission as a function of the location in the galaxy. López-Gonzaga et al. (2014), using IR interferometric observations with MIDI/VLTI, found that dust emission in the polar regions at scales of $5-10 \mathrm{pc}$ contributes four times more at $12 \mu \mathrm{m}$ than dust located in the torus. They also found a dependency on flux density as a function of the location along the north-south direction attributed to extension by the host galaxy. Our results using a solely 2D CLUMPY torus model and those by IR interferometric observations are of comparable order of magnitude, which tentatively indicates that the IR interferometric observations of NGC 1068 may be observing the dust emission from the optically thin dust of the torus.

\section{Spectral Decomposition}

Diffuse extended emission from dust and/or star formation regions surrounding the AGN can contribute at some level within the unresolved core of the SOFIA observations. What is the contribution of AGN emission within the unresolved core of our SOFIA observations?

We perform an SED analysis and fit the SED of the nuclear emission using the aperture and PSF-scaling photometric measurements, hereafter referred to as "large" and "small" aperture SEDs, respectively.

The large aperture SED is composed of our $10^{\prime \prime}$ aperture photometry $\left(F_{T}\right.$ in Table 1) in combination with Spitzer spectroscopic data taken from CASSIS $^{20}$ (Lebouteiller et al. 2011). In addition, we also include the $2-20 \mu \mathrm{m}$ photometry in a $4^{\prime \prime}(280 \mathrm{pc})$ aperture using the Mid-Infrared Test Observation System on the $8.2 \mathrm{~m}$ Subaru Telescope by Tomono et al. (2001). Figure 6 shows the nuclear SED using large (red dots) and small (black dots) apertures. It is worth noticing that (1) all the photometric measurements using large apertures are consistent with our aperture photometry, $F_{T}$, and (2) our $10^{\prime \prime}$ photometric measurement at $53 \mu \mathrm{m}$ shows an increase in flux density with respect to the $30-40 \mu \mathrm{m}$ photometric measurements, which indicates an extra emissive component at long wavelengths.

The large aperture SED was fitted as the contribution of the best inferred of the CLUMPY torus model to the small aperture SED (Section 3.1) and a star formation component. We use the CLUMPY torus model because it better reproduces the smaller

\footnotetext{
${ }^{20}$ The Combined Atlas of Sources with Spitzer IRS Spectra (CASSIS) is a product of the IRS instrument team, supported by NASA and JPL: http:// cassis.sirtf.com.
} 

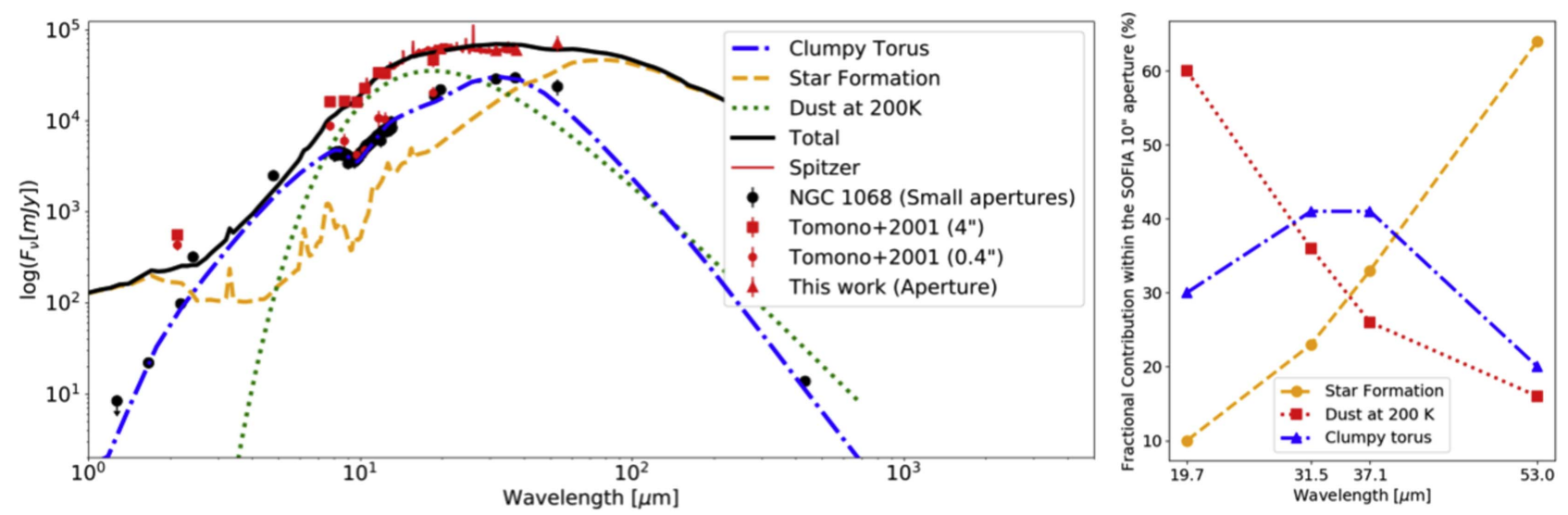

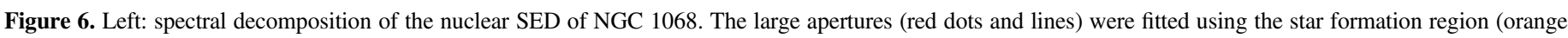

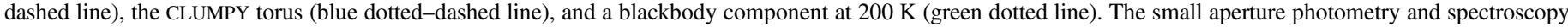

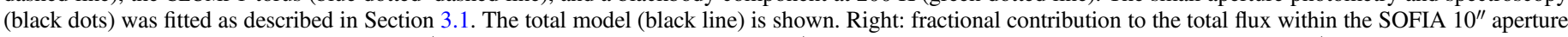
from the star formation (orange dashed line/circle), dust at $200 \mathrm{~K}$ (red dotted line/square), and CLUMPY torus (blue dotted-dashed line/triangle) components.

aperture SED than the smooth torus models. We use the empirical template of M82 as the star formation component from the Spitzer-space-telescope, Wide-field, InfraRed Extragalactic (SWIRE) template library ${ }^{21}$ (Polletta et al. 2007). We estimate the minimum reduced $\chi^{2}$, ensuring that the total model was within $10 \%$ of the measured total flux density of the large aperture SED. We find that the aperture photometric measurements in the $20-53 \mu \mathrm{m}$ wavelength range can be explained by the contribution of the torus emission and star formation region. However, we have to include an extra component to explain the excess of emission in the 8-20 $\mu \mathrm{m}$ wavelength range. This excess emission can be explained with the combination of the torus emission and an additional blackbody component with a characteristic temperature at $200 \mathrm{~K}$. We interpret the dust component at $200 \mathrm{~K}$ as dust emission arising from the NLR in the central $10^{\prime \prime}(700 \mathrm{pc})$ of NGC 1068 (see Tomono et al. 2001). Table 1 lists the fractional contribution of each component within the $10^{\prime \prime}$ aperture for the SOFIA observations. We estimate a 5\% uncertainty for the fractional contribution of each component shown in Table 1.

Based on our spectral decomposition within the $10^{\prime \prime}(700 \mathrm{pc})$ nuclear aperture, the fractional contribution to the total flux of the star formation increases with increasing wavelength, from $10 \pm 1 \%$ at $19.7 \mu \mathrm{m}$ to $64 \pm 3 \%$ at $53 \mu \mathrm{m}$ (Figure 6, right panel). The dust emission from extended dusty structures modeled as a blackbody component with a characteristic temperature at $200 \mathrm{~K}$ decreases with increasing wavelength, from $60 \pm 3 \%$ at $19.7 \mu \mathrm{m}$ to $16 \pm 1 \%$ at $53 \mu \mathrm{m}$. This extended emission, not associated with the torus, contributes $>80 \%$ of the total flux in the $8-20 \mu \mathrm{m}$ wavelength range and it is attributed to the N-S dust emission as seen by Bock et al. (2000) and also previously suggested by Cameron et al. (1993) and Mason et al. (2006). The fractional contribution to the total flux of the torus emission shows a turnover in the range of $30-40 \mu \mathrm{m}$ with a maximum fractional contribution to the total emission of $41 \pm 2 \%$, reaching a minimum of $20 \pm 1 \%$ at $53 \mu \mathrm{m}$.

\footnotetext{
$\overline{21}$ SWIRE templates can be found at http://www.iasf-milano.inaf.it/ polletta/ templates/swire_templates.html.
}

We can compare the potential contribution from the torus emission within the PSF-scaling photometry estimated in Section 2.3. In general, the fractional contribution of the total flux from the PSF-scaling method, percent PSF in Table 1, is slightly larger than the torus emission estimated by the spectral decomposition, percent Torus in Table 1. Specifically, the PSF-scaling method agrees with the torus emission within the PSF of SOFIA at all wavelengths within a fraction of $\sim 10 \%$ in the $20-53 \mu \mathrm{m}$ wavelength range. Based on Figure 6, the turnover of the torus emission in the range of $30-40 \mu \mathrm{m}$ can be distinguished from (a) the expected peak emission at $\sim 100 \mu \mathrm{m}$ by star formation regions, and (b) the extended dust emission associated with the NLR at shorter wavelengths.

\section{Conclusions}

Using SOFIA observations taken with FORCAST $(19.7-37.1 \mu \mathrm{m})$ and HAWC+ at $53.0 \mu \mathrm{m}$ on board SOFIA, we observationally find the turnover of the torus emission of NGC 1068 to be in the $30-40 \mu \mathrm{m}$ wavelength range. Specifically, the torus emission increases from 1 to $30 \mu \mathrm{m}$ and then we measure a decrease in the unresolved nuclear emission at $53 \mu \mathrm{m}$ with respect to the photometric measurements in the $30-40 \mu \mathrm{m}$ wavelength range. This result is in agreement with the observational constraint that the turnover does not occur until wavelengths $>31.5 \mu \mathrm{m}$ found by Fuller et al. (2016) using a sample of 11 Seyfert galaxies. Using CLUMPY torus models, we found a radius of $r_{\text {out }}=5.1_{-0.4}^{+0.4} \mathrm{pc}$ for the torus of NGC 1068. Our estimation of the torus size is in excellent agreement with the recently observed diameter of 7-10 pc by ALMA (Gallimore et al. 2016; García-Burillo et al. 2016; Imanishi et al. 2018). Although smooth torus models produce compatible results with those found by the CLUMPY torus models, they overestimate the nuclear SED in the FIR wavelength range. Despite the angular resolution of SOFIA, the 20-53 $\mu \mathrm{m}$ SOFIA observations together with (1) PSF-scaling and spectral decomposition techniques, and (2) CLUMPY torus models provide a tool to characterize the size of the torus in a large sample of AGNs when subarcsecond resolution observations by ALMA are not available.

We computed 2D images for the best inferred CLUMPY torus model using several SED coverages. We found that the 

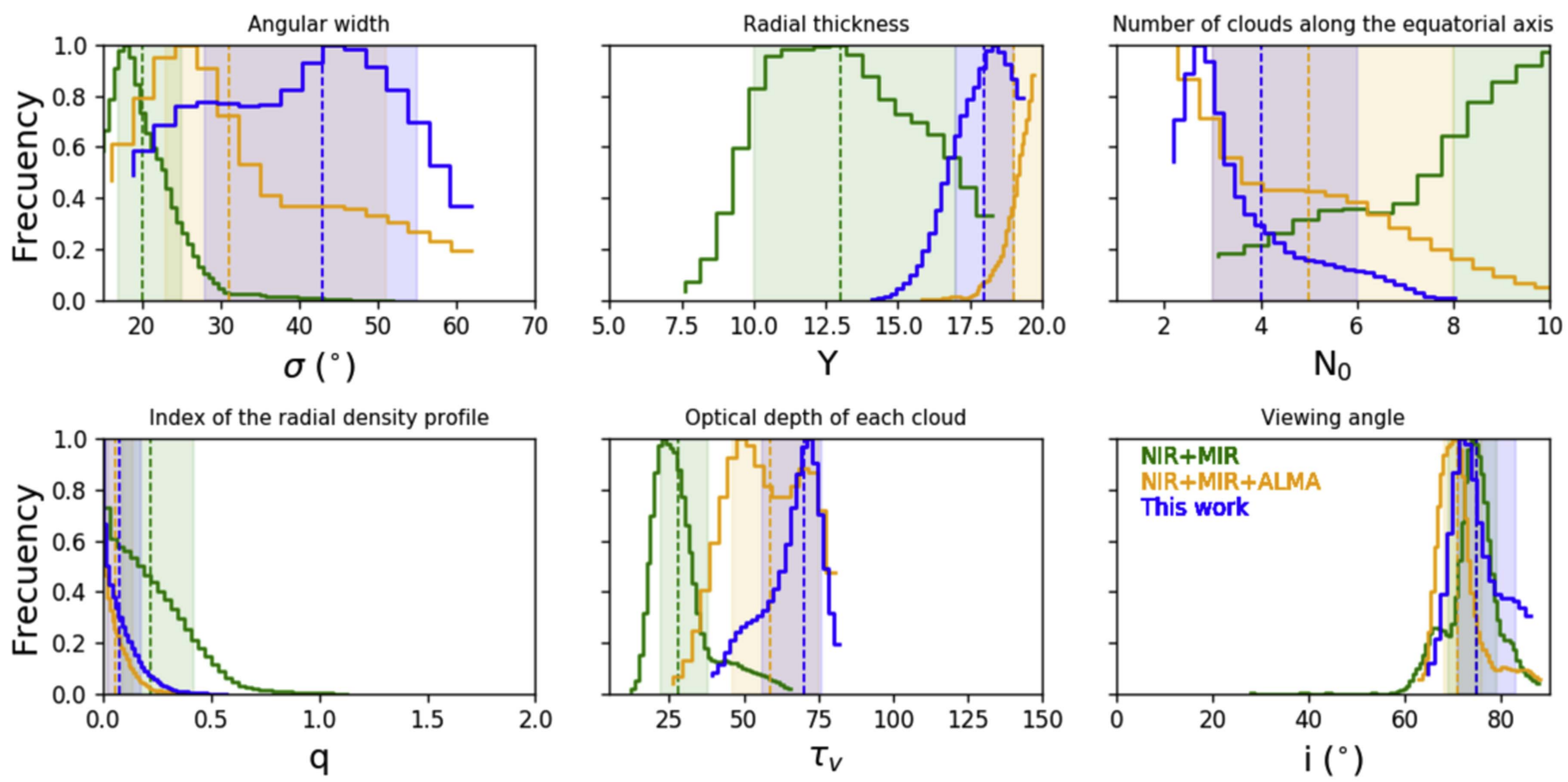

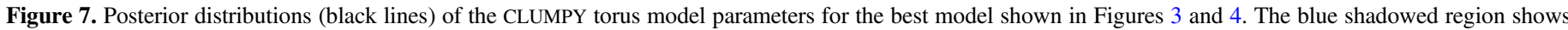
the $1-\sigma$ uncertainty and the blue line shows the best inferred model.

full extent and the cold dust of the torus are underestimated when a nuclear SED covering the $1-20 \mu \mathrm{m}$ wavelength range is used. The inferred CLUMPY torus from our $1-432 \mu \mathrm{m}$ nuclear SED reproduces well the ALMA observations. Specifically, the dust emission at $432 \mu \mathrm{m}$ is spatially coincident with the cloud distribution of the torus, while the morphology of the dust emission in the $1-20 \mu \mathrm{m}$ wavelength range probes mostly optically thin dust located above and below the equatorial plane of the torus. We estimated a contribution of the polar dust emission at $12 \mu \mathrm{m}$ to be $\sim 5$ times that from the central source, which indicates that the IR interferometric observations of NGC 1068 may be observing the dust emission from the optically thin dust of the torus.

Based on observations made with the NASA/DLR Stratospheric Observatory for Infrared Astronomy (SOFIA). SOFIA is jointly operated by the Universities Space Research Association, Inc. (USRA), under NASA contract NAS297001, and the Deutsches SOFIA Institut (DSI) under DLR contract 50 OK 0901 to the University of Stuttgart. Financial support for this work was provided by NASA through awards \#02_0035 and \#04_0048 issued by USRA. E.L.-R. acknowledges support from the Japanese Society for the Promotion of Science (JSPS) through award PE17783 and the National Observatory of Japan (NAOJ) at Mitaka and the Thirty Meter Telescope (TMT) Office at NAOJ-Mitaka for providing a space to work and great collaborations during the short stay at Japan. A.A.-H. acknowledges financial support from the Spanish Ministry of Economy and Competitiveness through grant AYA2015-64346-C2-1-P, which is party funded by the FEDER program. C.R.A. acknowledges the Ramón y Cajal Program of the Spanish Ministry of Economy and Competitiveness through project RYC-2014-15779 and the Spanish Plan Nacional de Astronomía y Astrofisíca under grant AYA2016-76682-C3-2-P.
Facilities: SOFIA(FORCAST, HAWC+).

Software: astropy (Astropy Collaboration et al. 2013).

\section{Appendix \\ CLUMPY Torus Model Paramater Posteriors}

Figure 7 shows the posterior distributions of the best inferred CLUMPY torus model shown in Figures 3 and 4.

\section{ORCID iDs}

Enrique Lopez-Rodriguez (1) https://orcid.org/0000-00015357-6538

Almudena Alonso-Herrero (ib https://orcid.org/0000-00016794-2519

Andreas Efstathiou (i) https://orcid.org/0000-0002-2612-4840

Kohei Ichikawa (i) https://orcid.org/0000-0002-4377-903X

Cristina Ramos Almeida (i) https://orcid.org/0000-00018353-649X

Dominic J. Benford (iD https://orcid.org/0000-0002-9884-4206

Ryan Hamilton (1) https://orcid.org/0000-0001-6350-2209

Fabio P. Santos (i) https://orcid.org/0000-0002-9650-3619

J. Staguhn (iD https://orcid.org/0000-0002-8437-0433

Terry Herter (i) https://orcid.org/0000-0002-3856-8385

\section{References}

Alonso-Herrero, A., Ramos Almeida, C., Mason, R., et al. 2011, ApJ, 736, 82 Asensio Ramos, A., \& Ramos Almeida, C. 2009, ApJ, 696, 2075

Asensio Ramos, A., \& Ramos Almeida, C. 2013, MNRAS, 428, 195

Astropy Collaboration, Robitaille, T. P., Tollerud, E. J., et al. 2013, A\&A, 558, A33

Barvainis, R. 1987, ApJ, 320, 537

Bland-Hawthorn, J., Gallimore, J. F., Tacconi, L. J., et al. 1997, Ap\&SS, 248, 9 Bock, J. J., Neugebauer, G., Matthews, K., et al. 2000, AJ, 120, 2904

Bohlin, R. C., Savage, B. D., \& Drake, J. F. 1978, ApJ, 224, 132

Cameron, M., Storey, J. W. V., Rotaciuc, V., et al. 1993, ApJ, 419, 136

Efstathiou, A., \& Rowan-Robinson, M. 1995, MNRAS, 273, 649

Emsellem, E., Fathi, K., Wozniak, H., et al. 2006, MNRAS, 365, 367 
Feltre, A., Hatziminaoglou, E., Fritz, J., \& Franceschini, A. 2012, MNRAS, 426, 120

Fritz, J., Franceschini, A., \& Hatziminaoglou, E. 2006, MNRAS, 366, 767

Fuller, L., Lopez-Rodriguez, E., Packham, C., et al. 2016, MNRAS, 462, 2618

Gallimore, J. F., Elitzur, M., Maiolino, R., et al. 2016, ApJL, 829, L7

García-Burillo, S., Combes, F., Ramos Almeida, C., et al. 2016, ApJL, 823, L12

Gratadour, D., Rouan, D., Grosset, L., Boccaletti, A., \& Clénet, Y. 2015, A\&A, 581, L8

Herter, T. L., Adams, J. D., De Buizer, J. M., et al. 2012, ApJL, 749, L18

Herter, T. L., Vacca, W. D., Adams, J. D., et al. 2013, PASP, 125, 1393

Hönig, S. F., Prieto, M. A., \& Beckert, T. 2008, A\&A, 485, 33

Ichikawa, K., Packham, C., Ramos Almeida, C., et al. 2015, ApJ, 803, 57

Imanishi, M., Nakanishi, K., Izumi, T., \& Wada, K. 2018, arXiv:1801.06564

Jaffe, W., Meisenheimer, K., Röttgering, H. J. A., et al. 2004, Natur, 429, 47

Kovács, A. 2006, PhD thesis, Caltech

Kovács, A. 2008, Proc. SPIE, 7020, 70201S

Lebouteiller, V., Barry, D. J., Spoon, H. W. W., et al. 2011, ApJS, 196, 8

López-Gonzaga, N., Jaffe, W., Burtscher, L., Tristram, K. R. W., \& Meisenheimer, K. 2014, A\&A, 565, A71

Lopez-Rodriguez, E., Packham, C., Jones, T. J., et al. 2015, MNRAS, 452, 1902

Lopez-Rodriguez, E., Packham, C., Roche, P. F., et al. 2016, MNRAS, 458, 3851
Mason, R. E., Geballe, T. R., Packham, C., et al. 2006, ApJ, 640, 612

Nenkova, M., Sirocky, M. M., Ivezić, Ž., \& Elitzur, M. 2008, ApJ, 685, 147

Packham, C., Young, S., Hough, J. H., Axon, D. J., \& Bailey, J. A. 1997, MNRAS, 288, 375

Polletta, M., Tajer, M., Maraschi, L., et al. 2007, ApJ, 663, 81

Raban, D., Jaffe, W., Röttgering, H., Meisenheimer, K., \& Tristram, K. R. W. 2009, MNRAS, 394, 1325

Ramos Almeida, C., Alonso-Herrero, A., Levenson, N. A., et al. 2014, MNRAS, 439, 3847

Ramos Almeida, C., Levenson, N. A., Alonso-Herrero, A., et al. 2011, ApJ, 731, 92

Ramos Almeida, C., \& Ricci, C. 2017, NatAs, 1, 679

Schartmann, M., Meisenheimer, K., Camenzind, M., Wolf, S., \& Henning, T. 2005, A\&A, 437, 861

Schinnerer, E., Eckart, A., Tacconi, L. J., Genzel, R., \& Downes, D. 2000, ApJ, 533,850

Scoville, N. Z., Matthews, K., Carico, D. P., \& Sanders, D. B. 1988, ApJL, 327, L61

Simpson, J. P., Colgan, S. W. J., Erickson, E. F., et al. 2002, ApJ, 574, 95

Tomono, D., Doi, Y., Usuda, T., \& Nishimura, T. 2001, ApJ, 557, 637

Vaillancourt, J. E., Chuss, D. T., Crutcher, R. M., et al. 2007, Proc. SPIE, 6678, $66780 \mathrm{D}$

Wittkowski, M., Kervella, P., Arsenault, R., et al. 2004, A\&A, 418, L39 